\title{
A Case of Unclassified Renal Cell Carcinoma Initially Considered as Translocation RCC and Review of Literature
}

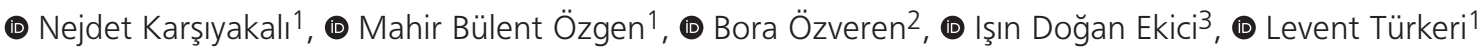 \\ ${ }^{1}$ Acıbadem Mehmet Ali Aydınlar University, Altunizade Hospital, Clinic of Urology, Istanbul, Turkey \\ ${ }^{2}$ Acıbadem Mehmet Ali Aydınlar University, School of Medicine, Altunizade Hospital, Clinic of Urology, Istanbul, Turkey \\ ${ }^{3}$ Acıbadem Mehmet Ali Aydınlar University, School of Medicine, Clinic of Pathology, Istanbul, Turkey
}

\begin{abstract}

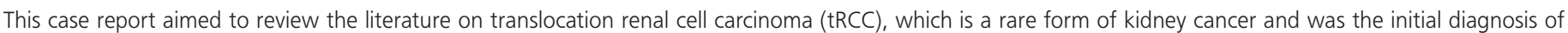

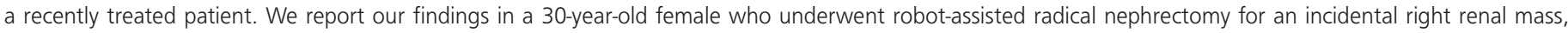


on current literature.
\end{abstract}

Keywords: Chromosome translocation, MiT family carcinomas, renal cell carcinoma, translocation renal cell carcinoma, Xp11 translocation

\section{Introduction}

Unclassified renal cell carcinoma (RCC) as defined by the 2016 World Health Organization (WHO) classification is a diagnostic category for renal tumours that do not fit into any of the wellrecognised subtypes $(1,2)$. Xp11 and $t(6 ; 11)$ translocation renal cell carcinomas (tRCC) are rare subtypes of RCC, which share variable morphological features that overlap considerably with other subtypes, including both clear cell (cCRCC) and papillary RCCs (pRCC) (2).

Xp11 and t(6;11) tRCCs have similar clinical, morphological, immunohistochemical and genetic features. Therefore, they are grouped as "MiT family translocation RCC" in the 2016 WHO urogenital tumour classification (1). Diagnosis of MiT family tRCC may pose some difficulties since $C C R C C$ and pRCC must be considered in differential diagnosis as they are more common in the adult age group (3).

$t(6 ; 11)$ tRCC generally has an indolent clinical behaviour, whereas Xp11 tRCC has a variable course. Rapid progressive disease and subsequent deaths have been reported in Xp11 tRCC (4). Nevertheless, the prognosis of the tRCCs remains unclear owing to its extremely low incidence rate, and patient series are limited in the literature often with short follow-up times. Surgery is the main treatment modality of localised disease. However, the most appropriate treatment option remains unclear for metastatic cases.
Thus, we present the details of the clinical, morphological and pathological features of tRCC in a 30-year-old female patient who underwent robot-assisted radical nephrectomy for an incidental right renal mass, which was initially considered as tRCC. Further molecular analysis categorised the tumour as unclassified RCC at the final pathologic evaluation.

\section{Case Presentation}

A 30-year-old female patient who had no chronic disease was referred to the urology outpatient department with a possible diagnosis of renal cancer according to magnetic resonance imaging (MRI) findings. The patient did not describe any history of macroscopic haematuria. She never smoked and had no occupational chemical exposure. Laboratory examination showed normal liver and renal function levels, and coagulation test findings were within normal limits. Complete blood count showed mild microcytic anaemia. No pathological findings were observed on the posteroanterior chest X-ray, which was performed according to the National Comprehensive Cancer Network guidelines. After gadolinium injection, minimal heterogeneous enhancement in arterial phase was detected on MRI (Figure 1). Contrast-enhanced computed tomography (CT) scan revealed a completely endophytic right renal mass at $42 \times 37 \times 40 \mathrm{~mm}$, located in the middle lower pole and displayed increased enhancement in the arterial phase (Figure 2). Although the renal mass was highly endophytic, robot-assisted

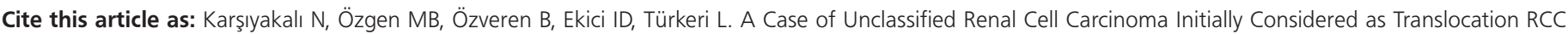
and Review of Literature. Bull Urooncol 2021;20(2):122-125 
partial nephrectomy was planned considering the patient's age. During the surgical planning, the patient was informed about the possibility of a radical nephrectomy since the mass was extending to the renal hilum resulting in close contact with the renal pelvis and major vessels.

An intraoperative ultrasound (US) examination via intracorporeal US probe confirmed a completely endophytic renal mass with possible invasion to the renal pelvis and close proximity to the anterior middle and lower branches of the renal artery and the posterior branch. Robot-assisted radical nephrectomy was performed since a partial nephrectomy required resection through the major vessels with a high risk of significant haemorrhage. The postoperative course was uneventful, and the patient was discharged on postoperative day 3 without complications.

Pathological examination revealed a pT1b renal tumour with the widest diameter of $4.3 \mathrm{~cm}$, Fuhrman grades 1-2 and negative surgical margins without coagulation necrosis, calcification, sarcomatoid features and microvascular invasion. Chromophobe RCC, hybrid renal tumours and MiT family RCCs were included in the differential diagnosis owing to the morphological features of the tumour.

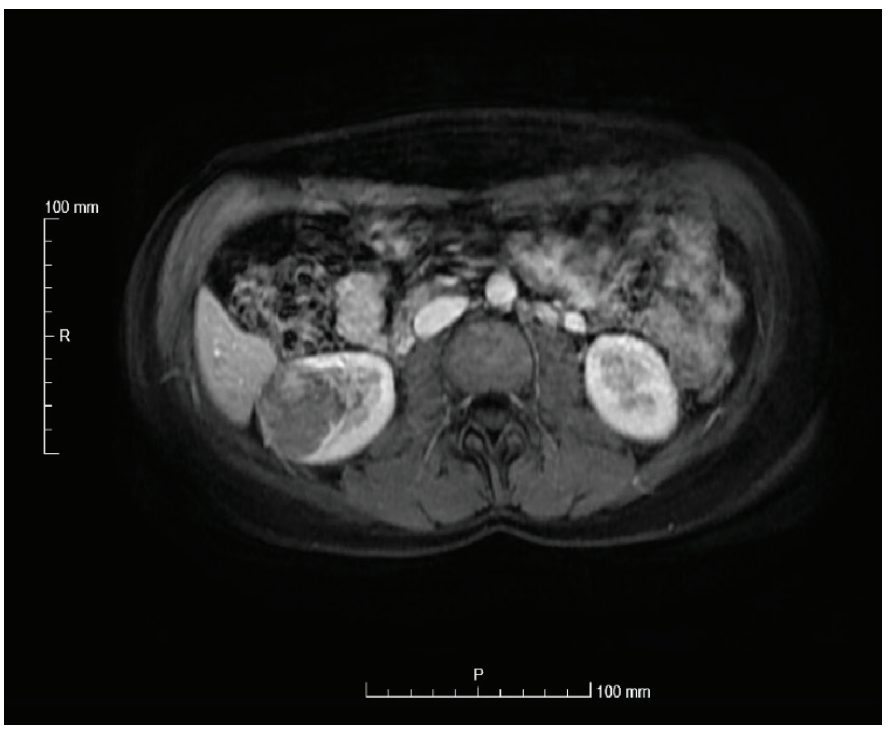

Figure 1. Magnetic resonance imaging findings of the right renal mass. Minimal heterogeneous contrast uptake is visualised on axial contrast-enhanced T1weighted images

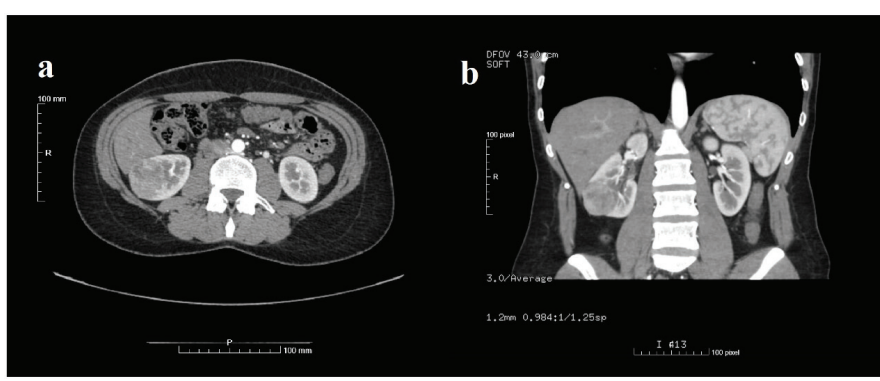

Figure 2. Contrast-enhanced computerised tomography findings of the right renal mass on axial (a) and coronal (b) sections. Highly endophytic renal mass without calcification is seen in the middle lower pole
Microscopic evaluation showed clear and eosinophilic cells with papillary features in a wide morphological spectrum (Figure 3a). Immunohistochemically, negative staining was observed for CK7, CD117, S-100, PAX8, Cytokeratin AE1/AE3 and Vimentin. However, mild positive staining was observed for TFE3 (Figure $3 \mathrm{~b}$ ). Based on these findings a preliminary diagnosis of tRCC was considered. However, further molecular characterisation with fluorescent in situ hybridisation (FISH) analysis failed to show any translocation, resulting in a final pathological diagnosis of unclassified RCC. The follow-up protocol was planned according to the European Association of Urology Guidelines on RCC recommendations for high-risk patients.

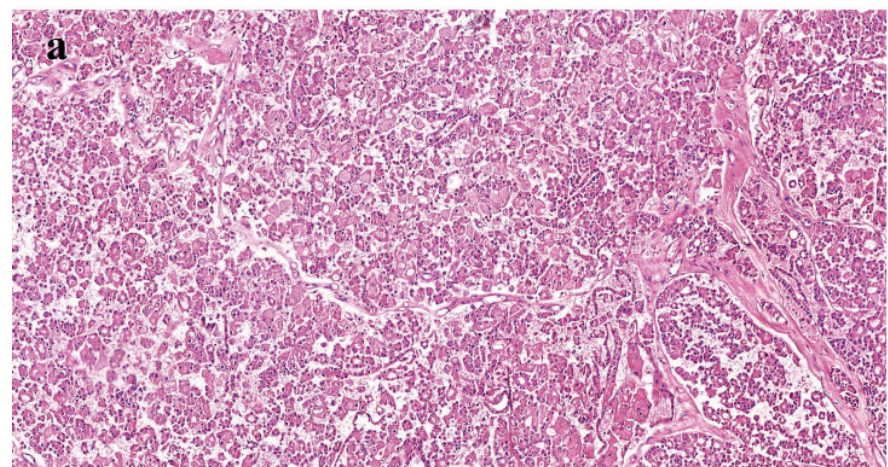

b

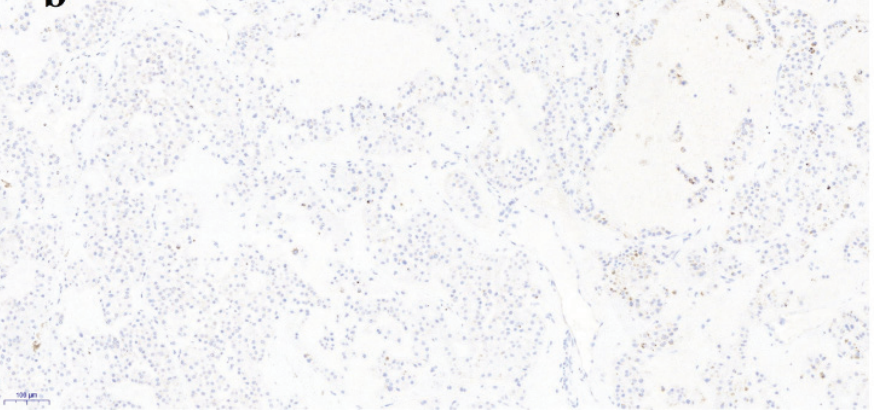

Figure 3. Clear and eosinophilic cells with papillary features in a wide morphological spectrum $(\mathrm{H} \& \mathrm{E}, \times 100)(\mathrm{a})$. Focal and weak positive brown staining was considered as uncertain (TFE3 immunohistochemistry, $\times 10$ ) (b) H\&E: Haemotoxylin and eosin

\section{Discussion}

Unclassified RCC comprise a significant proportion of adult renal epithelial tumours, accounting for $2-6 \%$ of RCCs (2). Unclassified RCC remains a diagnosis of exclusion, with careful characterisation of recognizable histologic subtypes through immunohistochemistry and molecular analysis if necessary. MiT family tRCC is a subtype that must be considered. Similarly, in the present case, the diagnosis of unclassified RCC had been possible after the elimination of the tRCC by FISH. Estimated tRCC incidence was reported to be approximately $4.2 \%$ (5). The rate of diagnosis is arguably low due to the morphological similarities with other more frequent RCC types more commonly seen in adults such as cCRCC and pRCC (6). tRCCs can be diagnosed by pathological evaluation of the excised renal 
mass. The renal mass was detected incidentally in our patient by imaging modalities due to symptoms not related to the genitourinary system, and a definitive diagnosis of tRCC was designated after pathological examination.

It has been reported that on CT imaging, both high attenuation areas [>40 Hounsfield Unit (HU)] due to high cellular component and heterogeneous low attenuation areas due to necrosis/ haemorrhage can be detected simultaneously (7). Similar to these reports, we observed both high attenuation (up to 80 $\mathrm{HU}$ ) and low attenuation areas together on CT imaging in the present case.

Urologists must be familiar with tRCC, since they are seen in younger patients and may have aggressive clinical behaviour. Aggressive behaviour defined as metastatic potential was reported to be $46 \%$ and $17 \%$ in Xp11 and $t(6 ; 11)$ tRCCs, respectively (8). Age, gender and Fuhrman grade had no significant effect on aggressive clinical behaviour. In contrast, high tumour diameter, existence of necrosis and $>50 \%$ immunohistochemical staining with MET were statistically significantly correlated with aggressive clinical behaviour (8).

Xp11 tRCC and ccRCCs are extremely macroscopically similar, as they are both brown-yellowish in colour and may have necrotic and haemorrhagic areas (9). Microscopically, clear epithelioid cells with papillary structure are the most remarkable features of the tRCCs. Similarly, clear and eosinophilic cells with papillary features were seen in the present case. Both tRCC types may have variable morphological features such as solid, nested, alveolar, tubulocystic and papillary growth as well as pseudocapsules, hyalinisation, necrosis and psammoma bodies $(6,8)$. Therefore, MiT family tRCCs can be confused with ccRCC and pRCC (3).

Immunohistochemical diagnostic methods are important for differential diagnosis. In this context, cathepsin $\mathrm{K}$ is a significant immunohistochemical marker, which always stains negative in other RCC subtypes. In contrast, positive staining is observed in approximately $60 \%$ of the Xp11 and almost all of the $t(6: 11)$ tRCC cases (10). The most sensitive and specific immunohistochemical marker for Xp11 tRCC is strong nuclear TFE3 immunoreactivity (6). Immunohistochemical staining with TFE3 was seen in all of the Xp11 tRCCs although with variable staining intensity (11). Similarly, there was mild positive staining with TFE3 in our case with immunohistochemistry. The use of a narrow immunohistochemical panel when making a differential diagnosis for RCC in daily practice may lead to false results (8). In the immunohistochemical differential diagnosis of these cases, staining negative with CK7 and positive with cathepsin $\mathrm{K}$ suggested Xp11 tRCC $(3,8)$. Calio et al. (8) defined a useful immunohistochemical panel that included cathepsin K, CD68, CK7, CA9 and PAX8 and excluded CD10 and AMACR.

Since the MiT family tRCCs may display a highly variable morphology, it is not always possible to diagnose with immunohistochemical examinations. Analysis of genetic changes by FISH is the gold standard technique in these cases and makes it possible to evaluate the TFE3 and TFEB genes (12). In evaluation with FISH, TFE3 fluorescent signal was observed in $45-90 \%$ and $61-94 \%$ of the cells in Xp11 and t(6;11) tRCC, respectively (8). Moreover, higher fluorescent signal with TFE3 in Xp11 tRCC, higher TFEB/VEGFA gene copy number and amplification were reported to be associated with the aggressive behaviour of the tumour (8).

Few studies evaluated VEGFR-targeted agents in metastatic tRCCs and reported objective response rates of up to $30 \%$. Progression-free survival was (PFS) 7.1-8.2 months in these series (13). Reported PFS was four, three and four months in metastatic Xp11 tRCC cases treated with sunitinib, sorafenib and temsirolimus, respectively (11). In a retrospective study of 24 patients treated with immune check-point inhibitors as a second or subsequent line treatment for metastatic tRCC, PFS was 2.5 (1-40) months, and partial response was observed in 4 (16.7\%) patients and stable disease in $3(12.5 \%)$ (14). A recent study with cabozantinib, which is a multiple tyrosine kinase inhibitor (VEGFR, MET and AXL), was found to be effective and associated with downregulation of cathepsin K in tRCC (15).

\section{Conclusion}

Although, tRCCs are rare RCC subtypes, they may present a diagnostic problem. Definitive diagnosis is possible after surgical resection of the tumour by immunohistochemical analysis. If the diagnosis remains unclear after immunohistochemical examinations, genetic analyses can be performed with FISH.

\section{Acknowledgements}

Publication: The results of the study were not published in full or in part in form of abstracts.

Contribution: There is not any contributors who may not be listed as authors.

Conflict of Interest: No conflict of interest was declared by the authors.

Financial Disclosure: The authors declared that this study received no financial support.

\section{Ethics}

Informed Consent: The patient was informed about the possibility of a radical nephrectomy since the mass was extending to the renal hilum resulting in close contact with the renal pelvis and major vessels.

Peer-review: Externally peer-reviewed.

\section{Authorship Contributions}

Concept: B.Ö., L.T., Design: B.Ö., L.T., Data Collection or Processing: N.K., I.D.E., Analysis or Interpretation: N.K., M.B.Ö., I.D.E., Literature Search: N.K., Writing: N.K.,

\section{References}

1. Moch H, Cubilla AL, Humphrey PA, et al. The 2016 WHO classification of tumours of the urinary system and male genital organs-part a: renal, penile, and testicular tumours. Eur Urol 2016;70:93-105.

2. Sirohi D, Smith SC, Agarwal N, et al. Unclassified renal cell carcinoma: diagnostic difficulties and treatment modalities. Res Rep Urol 2018;10:205-217.

3. Ross H, Martignoni G, Argani P. Renal cell carcinoma with clear cell and papillary features. Arch Pathol Lab Med 2012;136:391-399.

4. Calio A, Segala D, Munari E, et al. MiT family translocation renal cell carcinoma: from the early descriptions to the current knowledge. Cancers (Basel) 2019;11:1110. 
5. Zhong M, De Angelo P, Osborne L, et al. Dual-color, break-apart FISH assay on paraffin-embedded tissues as an adjunct to diagnosis of Xp11 translocation renal cell carcinoma and alveolar soft part sarcoma. Am J Surg Pathol 2010;34:757-766.

6. Argani P. MiT family translocation renal cell carcinoma. Semin Diagn Pathol 2015;32:103-113.

7. Koo HJ, Choi HJ, Kim MH, et al. Radiologic-pathologic correlation of renal cell carcinoma associated with Xp11.2 translocation. Acta Radiol 2013;54:827-834.

8. Calio A, Brunelli M, Segala D, et al. Comprehensive analysis of 34 MiT family translocation renal cell carcinomas and review of the literature: investigating prognostic markers and therapy targets. Pathology 2020;52:297-309.

9. Argani P, Lae M, Ballard ET, et al. Translocation carcinomas of the kidney after chemotherapy in childhood. J Clin Oncol 2006;24:1529-1534.

10. Martignoni G, Gobbo S, Camparo P, et al. Differential expression of cathepsin $\mathrm{K}$ in neoplasms harboring TFE3 gene fusions. Mod Pathol 2011;24:1313-1319.
11. Su HH, Sung MT, Chiang PH, et al. The preliminary experiences of translocation renal cell carcinoma and literature review. Kaohsiung I Med Sci 2014;30:402-408.

12. Rao Q, Williamson SR, Zhang S, et al. TFE3 break-apart FISH has a higher sensitivity for $\mathrm{Xp11.2}$ translocation-associated renal cell carcinoma compared with TFE3 or cathepsin $\mathrm{K}$ immunohistochemical staining alone: expanding the morphologic spectrum. Am J Surg Pathol 2013;37:804-815.

13. Choueiri TK, Lim ZD, Hirsch MS, et al. Vascular endothelial growth factor-targeted therapy for the treatment of adult metastatic Xp11.2 translocation renal cell carcinoma. Cancer 2010; 116:5219-5225.

14. Boileve A, Carlo MI, Barthelemy $\mathrm{P}$, et al. Immune checkpoint inhibitors in MITF family translocation renal cell carcinomas and genetic correlates of exceptional responders. J Immunother Cancer 2018;6:159.

15. Martinez Chanza N, Xie W, Asim Bilen M, et al. Cabozantinib in advanced non-clear-cell renal cell carcinoma: a multicentre, retrospective, cohort study. Lancet Oncol 2019;20:581-590. 